\title{
El aprendizaje significativo de la segunda ley de la termodinámica*
}

\section{José Otero**}

Universidad de Alcalá de Henares

\section{Isabel Brincones}

\author{
Universidad Autónoma de Madrid
}

La organización de la estructura conceptual del currículum de ciencias, en cualquier nivel, se ve afectada por dos tipos de condiciones. Las primeras se relacionan con la naturaleza de la disciplina; son los criterios llamados «lógicos» que limitan el tipo de relaciones que se pueden establecer entre conceptos. Si el concepto de «fuerza electromotriz», por ejemplo, se introduce a partir de los conceptos de «trabajo» o «energía» y «carga eléctrica» éstos. deberán relacionarse a través de un cociente.

Existen, además, criterios llamados "psicológicos», relacionados con la persona que aprende, que condicionan también la elección de una u otra clase de organización conceptual del currículum. Es posible que la presentación inicial del concepto de fuerza electromotriz a través de una definición como cociente entre la energía que pasa de la forma no eléctrica a la forma eléctrica por unidad de carga, sea inapropiada para alumnos en cursos introductorios de física. Los criterios psicológicos aconsejarían, por ejemplo, el uso de símiles mecánicos que activasen esquemas poseídos por el alumno, u otras posibles reorganizaciones del contenido conceptual para lograr un mejor aprendizaje.

Las preconcepciones de los alumnos sobre los fenómenos estudiados, particularmente los fenómenos naturales, son otra de las fuentes de la que se desprenden criterios psicológicos para la organización del currículum. Los alumnos inician el aprendizaje de las ciencias con ideas previas que deben tenerse en cuenta si se quiere evitar que interfieran de manera indeseable. Por tanto, un diseño adecuado del contenido conceptual del currículum de ciencias estriba en la coordinación de los dos tipos de criterios que se acaban de mencionar: los que dependen de la forma en que aprende el alumno y los relacionados con la naturaleza de la ciencia.

* El trabajo que recoge este artículo constituye en realidad un estudio exploratorio realizado dentro de un proyecto de investigación subvencionado por el C.I.D.E. Agradecemos la colaboración de los profesores que utilizaron los materiales de enseñanza en sus aulas: Juan García Quintana, del I. B. «Ramiro de Maeztu»; Ricardo Garrote, Paloma Montes y Cristina Tejel, del I. B. «Herrera Oria», ambos de Madrid. Hacemos extensivo nuestro agradecimiento al jefe del Seminario de Física y Química de este último centro, Francisco L. Rupérez. Damos las gracias también a Miguel Ramos, profesor de la Universidad de Alcalá, por la ayuda prestada.

** Dirección de los autores: Departamento de Física. Universidad de Alcalá. Alcalá de Henares (Madrid). 
En lo que respecta a los criterios lógicos, el currículum de ciencias, especialmente en los niveles secundario y terciario, ha sido tradicionalmente estructurado de manera que refleja una visión «estática» del conocimiento científico. La ciencia que presentan los cursos de estos niveles está caracterizada por estructuras conceptuales estables y definitivas, presentadas sin dar cuenta del proceso mediante el cual se ha llegado a establecerlas.

Esta visión de la ciencia se acompaña frecuentemente por un enfoque empirista del conocimiento: la correspondencia entre realidad y conocimiento parece directa y unívoca, y los conceptos y principios se presentan fundamentados en la experiencia, en los datos, por medio de un proceso de inferencia inductiva. En 1970, ya se explicaba esta concepción de la enseñanza de la ciencia:

Hasta los días del primer Sputnik, la tradición en la enseñanza de la ciencia era atiborrar al alumno con cantidades enormes de información sobre hechos «objetivos» y leyes de la naturaleza «probadas»... Esta clase de enseñanza de la ciencia se aceptaba como el mejor de los métodos desde los años 1870 , y era una aplicación de la filosofía de la ciencia inductivista-realista: el mundo era real y las mismas leyes, absolutamente ciertas, lo describían tal como era. Todos los hechos eran objetivos y pertenecían al tesoro positivo de la ciencia. Algunas teorías habían sido probadas más allá de cualquier duda, principalmente como consecuencia de una paciente recolección de datos. (Elkana, 1970, pág. 17.)

En los años 60 se produjeron en los países anglosajones los primeros síntomas de reacción a esta concepción de la ciencia que, en ciertos aspectos, se manifestaba tanto en los círculos filosóficos como en los niveles de educación más elementales. Científicos y filósofos como Conant, Feyerabend, Holton, Kuhn, Lakatos, etc., protagonizaron este movimiento de renovación. Hasta aquel momento, como apunta $\mathrm{S}$. Toulmin refiriéndose al trabajo de Kuhn,

... la filosofía americana de la ciencia había estado dominada durante 40 años por un empirismo lógico autosuficiente y antihistórico que había sido heredado del Círculo de Viena, y una virtud real de su trabajo fue poner énfasis en la necesidad de un enfoque de la ciencia más histórico y menos formalista. (Toulmin, 1972, pág. 126.)

Elkana explica la transformación que sufrió la concepción filosófica de la ciencia:

En los últimos veinte años ha estado surgiendo una nueva filosofía de la ciencia y ha dejado atrás el positivismo ahistórico o incluso antihistórico... De acuerdo con esta filosofía, el intento de describir el lenguaje o método de la ciencia no es más que un ejercicio lógico, valioso como tal, pero que no tiene nada que ver con la ciencia en realidad... para mostrar que hay muchos lenguajes diferentes de la ciencia, esta filosofía usa dos clases de argumentos: un argumento histórico y un argumento psicológico. Estas dos clases de argumentos se introducen en lugar del argumento lógico que se aplica solamente al producto terminado y bien formulado, resultado de la investigación científica. La lógica de la ciencia es solamente la descripción de la ciencia ideal pulida, formulada axiomáticamente; la lógica no puede dar cuenta de la ciencia como proceso, de la ciencia en desarrollo (op. cit., pág. 24).

De esta forma el estudio filosófico de la ciencia tiende a cambiar su foco de atención de los análisis lógicos «estáticos» sobre productos terminados, característicos del positivismo lógico, al estudio de la cien- 
cia en desarrollo, sobre todo en sus aspectos sociales e históricos (más que en el terreno de la producción individual).

Este cambio tiene implicaciones en el campo de la enseñanza. El diseño de la estructura conceptual del currículum requiere una selección y ordenación de los conceptos de la disciplina científica de que se trate. Según se acaba de indicar, este análisis ha permanecido tradicionalmente dentro del «contexto de justificación» (Reichenbach, 1938). El currículum se estructura de acuerdo con las relaciones lógicas que existen entre conceptos constituyentes de teorías «terminadas». El resultado fue, con mucha frecuencia, convertir las asignaturas científicas en lo que Schwab ha llamado «retórica en torno a conclusiones»:

[La ciencia] Se enseña casi completamente como «retórica en torno a conclusiones» en la cual las construcciones actuales y temporales del conocimiento científico se transmiten como verdades empíricas, literales e irrevocables (Schwab, 1966, pág. 24).

En el trabajo que sigue a continuación se apuntan algunos problemas que surgen como consecuencia del fundamento epistemológico del currículum de ciencias que se acaba de explicar y que son predecibles a partir de la teoría de la asimilación de Ausubel y Novak. Estos problemas están relacionados con la arbitrariedad de las ideas que se presentan al estudiante:

La esencia del proceso de aprendizaje significativo es que las ideas expresadas simbólicamente se relacionen de manera no arbitraria y sustantiva (no al pie de la letra) con lo que el alumno ya sabe. (Ausubel, Novak y Hanesian, 1978, pág. 41; subrayado añadido.)

Una organización como la que se ha descrito parece producir relaciones con lo que el alumno ya sabe que son intrínsecamente arbitrarias.

El primer tipo de arbitrariedad que parece propiciar la organización que se ha descrito, se relaciona con la ausencia de problemas en el contenido conceptual del currículum. Los conceptos, a lo largo de la historia de la ciencia, no se introducen simplemente como posibilidades lógicas que sirven para engrosar el contenido de una disciplina, sino como respuesta a algún problema extraordinario (Toulmin, $o p$. cit., págs. 207-208). Sin embargo, en los libros de texto y en los cursos de ciencias se le dan al alumno fundamentalmente respuestas, en forma de conceptos y principios explicativos, y rara vez se le presentan los problemas que los originaron. El problema, que surgió como una discrepancia entre las observaciones y las expectativas del investigador, y que motivó las respuestas conceptuales está normalmente ausente; es difícil que el alumno pueda identificarlo a través de los conceptos y principios que se le ofrecen. Los conceptos, por tanto, en expresión de Lakatos, «caen del cielo» ${ }^{1}$.

Una segunda clase de arbitrariedad está relacionada directamente con la fundamentación de los conceptos y explicaciones conceptuales que se le of recen al estudiante.

La generación de conceptos y principios se explica, de la forma más adecuada, considerándolos como construcciones que resultan de la interrelación entre los datos empíricos y el marco conceptual que ya posee el investigador. Los conceptos científicos se forman a lo largo de un estadio psicogenético, de producción en la mente del científico crea- 
dor y, más adelante, un proceso evolutivo en el que son conformados por la influencia de la comunidad científica (un estadio sociogenético).

Esta evolución no aparece en el enfoque empirista usado con frecuencia en cursos y libros de texto. Se le presentan al estudiante construcciones artificiales cuya única justificación estriba, aparentemente, en los fenómenos que explican, ocultando el proceso que se acaba de mencionar. Sin embargo, los hechos por sí mismos no pueden determinar el conocimiento conceptual necesario para explicarlos.

Efectos de la organización conceptual «estática» del currículum de ciencias: hipótesis del trabajo

Con objeto de explorar la importancia de los problemas anteriores en la práctica educativa, se llevó a cabo un estudio exploratorio (no tiene carácter experimental) mediante el que se investigaron los efectos de organizaciones alternativas del contenido conceptual del currículum, de acuerdo con los criterios que se acaban de exponer. Se utilizaron dos organizaciones conceptuales diferentes de una lección sobre el teorema de Carnot y la Segunda Ley de la termodinámica dirigida a alumnos de último año de bachillerato o C.O.U.

Las hipótesis del trabajo son las siguientes:

a) Una organización conceptual que, además de presentar conceptos en su forma final, ponga también de manifiesto los problemas que propiciaron su generación, facilitará la no arbitrariedad y, por tanto, el aprendizaje significativo.

b) Una organización conceptual que, además de presentar conceptos en su forma final, ponga también de manifiesto la evolución que han sufrido históricamente estos conceptos y, por tanto, la influencia del marco conceptual general en el que se han desarrollado, facilitará la no arbitrariedad y, por tanto, el aprendizaje significativo.

Los resultados del estudio proporcionan algunas indicaciones que concuerdan con las hipótesis que se acaban de plantear, aunque, estrictamente, deben tomarse como sugerencias para trabajos experimentales, posteriores, de mayor precisión. Otros resultados de interés remiten a problemas de aprendizaje más fundamentales que los que se han mencionado y que no habían sido contemplados inicialmente en el estudio.

\section{Procedimiento}

Se seleccionaron cuatro clases de tercero de B.U.P. (alumnos de 17 años) en dos Institutos de Madrid capital. En dos grupos (GE1, GE2) se enseñó la Segunda Ley de la termodinámica usando los materiales experimentales que se describen más adelante, durante un período de tres semanas. En los otros dos grupos se usaron materiales tradicionales, que se describen también a continuación, durante el mismo tiempo. Todos los grupos tenían profesores diferentes. Por dificultades en el calendario de realización de las pruebas finalmente sólo se pudo disponer de las contestaciones de uno de los grupos que usaron materiales tradicionales (GT). Se sometió también a un grupo de alumnos de primer curso de universidad (GU) a una de las pruebas finales utilizadas en el trabajo. Estos alumnos habían seguido, de manera totalmente independiente, un curso de introducción a la Mecánica y la Termodinámica, dentro de la licenciatura en Ciencias Químicas. 
Todos los alumnos de los grupos GE y GT recibieron materiales escritos sobre la Segunda Ley de la Termodinámica. Los materiales experimentales consistian en un documento de 40 páginas en el que se organizaba el contenido de acuerdo con las orientaciones que se acaban de exponer:

a) La estructura de alto nivel de los materiales refleja una organización "problema/solución» (Meyer et al., 1984). Es decir, el principio fundamental que se pretende enseñar a los alumnos (el enunciado de Kelvin-Planck de la Segunda Ley de la Termodinámica) se presenta como solución a un problema planteado previamente: la mejora del rendimiento de las máquinas térmicas. Existen otros conceptos clave que, de igual modo, se presentan como respuesta a un problema científico previo.

b) Los principios y conceptos clave que se introducen como respuesta a estos problemas no aparecen determinados solamente por los datos experimentales, sino también por ideas pertenecientes al marco conceptual general del científico o de la comunidad científica en cada época.

El propósito es lograr estructuras conceptuales con mayor significatividad potencial: se intenta explicar la génesis de conceptos y principios que se presentan como resultados obvios en las organizaciones tradicionales del conocimiento científico.

Un diagrama de la organización conceptual de los materiales experimentales puede verse en la Figura 1.

\section{FIGURA 1}

Organización conceptual de los materiales experimentales.

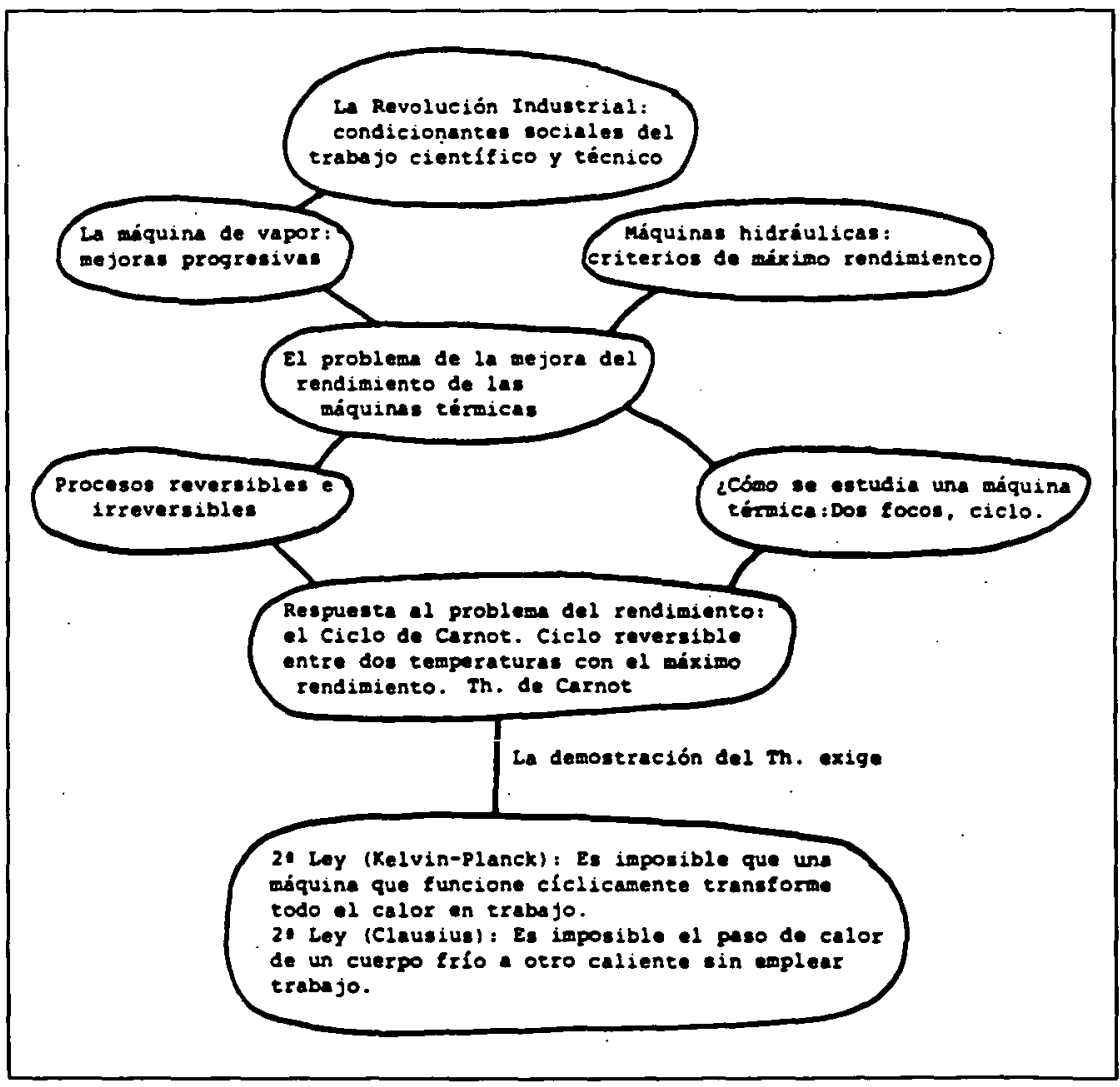


Los materiales que estaban organizados de manera tradicional consistían en una copia de parte del capítulo que Halliday y Resnick (1977) dedican a la Segunda Ley, a la que se añadió un pequeño apartado (demostración del Teorema de Carnot a partir de la Segunda Ley) para lograr que los conceptos y principios básicos fuesen los mismos en todos los grupos. Las ideas incluidas y su organización, que puede considerarse representativa de los enfoques tradicionales, se muestran en la Figura 2.

FIGURA 2

Organización conceptual de los materiales tradicionales.

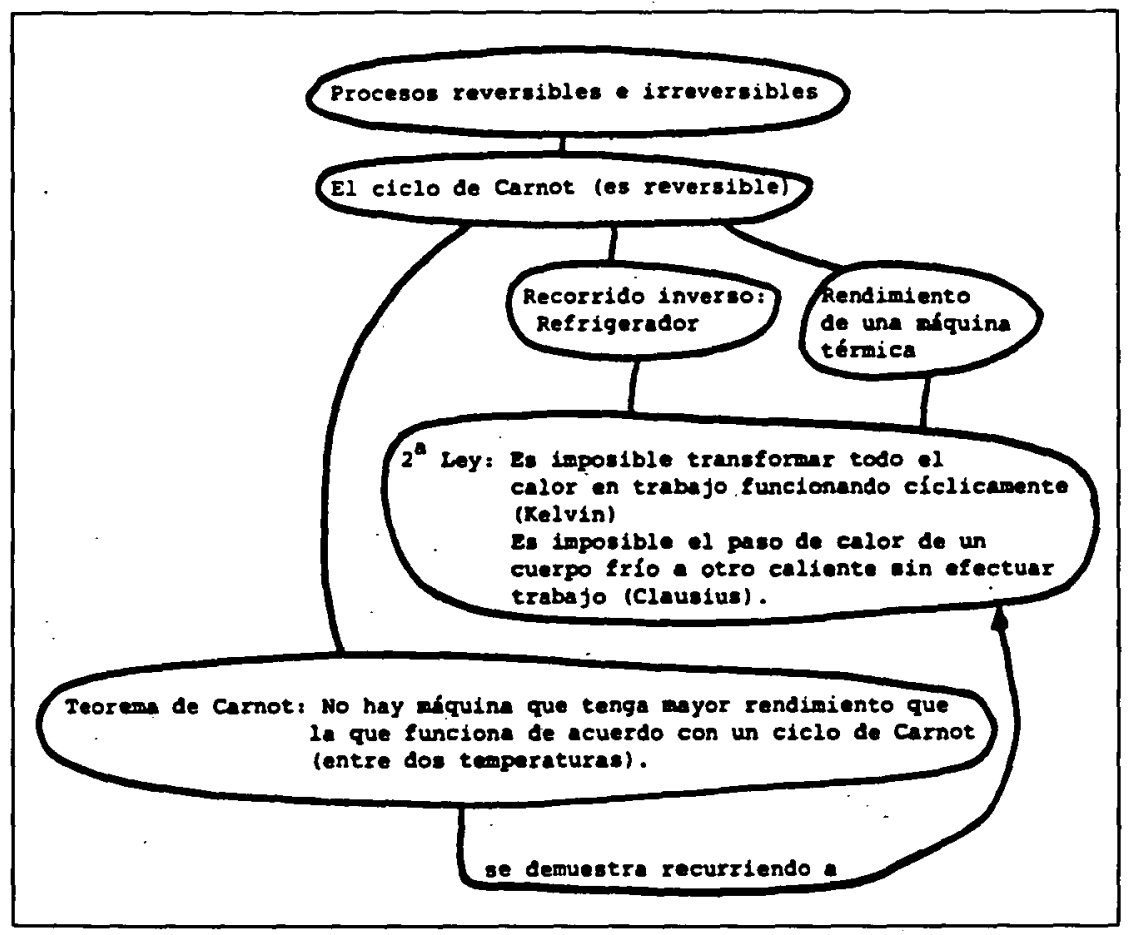

Se evaluó a los alumnos de diversas formas: mediante pruebas escritas de respuesta abierta y de elección Verdadero/Falso; también se le propusierơn preguntas sobre una siruación problemática, en relación con la Segunda Ley, a grupos de 3-4 alumnos y se grabó la discusión que mantenían para contestar a ellas. La evaluación se llevó a cabo al finalizar el tratamiento, en febrero (prueba $A$ ), y 9 semanas más tarde, en mayo (prueba B), con el fin de medir el recuerdo y, por tanto, la fortaleza de las conexiones entre el nuevo material presentado y lo que ya sabían los alumnos.

\section{Resultados y discusión}

Los resultados más importantes que se obtienen se sintetizan a continuación. Denotan, por una parte, el efecto que tienen las preconcepciones sobre el aprendizaje y, por otra, la influencia de la organización conceptual general, variable sobre la que se centraba nuestro interés:

I) Aparecen concepciones erróneas (desde el punto de vista de la ortodoxia científica), o preconcepciones, que interfieren con el apren- 
dizaje de los conceptos científicos y que afectan casi por igual a estudiantes de los grupos «experimentales» y «tradicionales». Los estudiantes recurren con frecuencia a estas concepciones para dar explicaciones.

II) Los estudiantes repiten en ocasiones, al dar sus explicaciones, los errores cometidos por los científicos a lo largo de la historia.

III) A los estudiantes les resulta difícil captar la estructura conceptual general, de alto nivel (macroestructura), de los materiales.

IV) En ocasiones se encontró que la presentación al estudiante de la génesis de conceptos y principios mejoraba el aprendizaje significativo. En otros casos no se encontraron diferencias con la presentación tradicional.

V) No se pudieron encontrar diferencias en el aprendizaje significativo de la segunda ley, debidas a la estructura problema-solución.

\section{I) Interferencia de las concepciones erróneas}

Ideas distintas de las presentadas en clase de física (podrían llamarse de sentido común) interfirieron con el aprendizaje de nuevos conceptos. El efecto de esta interferencia se dejó sentir para los estudiantes en los tres grupos. Ejemplos de este tipo de ideas son las siguientes:

a) «Posibilidad teórica de un rendimiento del 100 por 100 en una máquina térmica»:

Aun cuando la Segunda Ley de la Termodinámica lo prohíbe, después del estudio de los materiales se detectó la existencia de un número de estudiantes que creían que era posible tener una máquina de 100 por 100 de rendimiento en la teoría, aunque sea imposible en la práctica. Ejemplos de esta creencia son las siguientes contestaciones a una de las preguntas formuladas:

Pregunta A7.

En las máquinas térmicas, como las de vapor, se pierde una cierta cantidad de calor que se disipa en la atmósfera. Se ha diseñado un motor de vapor que posee todo él una doble pared por donde se hace circular agua fría. Regulando el flujo de agua por la doble pared se puede conseguir que las pérdidas de calor hacia el exterior sean mínimas y que todo el calor, que de otra manera se perdería, pase al agua. El agua se envía después a la caldera y sirve para producir más vapor que hace mover la máquina. El rendimiento de esta manera, según aseguran los constructores puede llegar a aproximarse al 100 por 100 , es decir, todo el calor que proporciona el combustible se convierte en trabajo.

a) ¿Crees que esto es posible en la práctica? ¿Por qué?

b) ¿Crees que esto es posible en teoría? ¿Por qué?

No, sería imposible en la práctica ya que toda máquina térmica debe funcionar entre dos temperaturas... En teoría sí es posible ya que teóricamente se puede conseguir un 100 por 100 de rendimiento de la máquina (GE1, A, 35).

No [sería posible en la práctica] porque a pesar de todo el calor del agua se perdería, a veces como energía interna o en el recorrido... En la teoría es realizable aunque creo que presenta muchas dificultades (GE2, A, 33).

Pienso que no es posible [en la práctica] puesto que según el teorema de Carnot, no existe ninguna máquina capaz de producir todo el calor en trabajo... En teoría puede que sí porque según lo propuesto el agua no se enfría (GT, A, 31).

En la Tabla 1 se recogen las contestaciones dadas por los alumnos a la pregunta $A 7$. 

máquina con el $100 \%$ de rendimiento (Preg. A7).

\begin{tabular}{lccc}
\hline Grupos & GT & GE1 & GE2 \\
\hline Contestaciones & & & \\
Correcta: Imposible en la pa y & & 26 & 14 \\
en la ta & 16 & 9 & 13 \\
Errónea: Imposible en la pa no & & 3 & 7 \\
en la ta: & 10 & 38 & 34 \\
Otras/NC & 7 & 33 & \\
Total & 33 & \\
\hline
\end{tabular}

No existen diferencias significativas $(p<0,05)$ en los porcentajes de respuestas erróneas tanto entre los GE y el GT como entre GE1 y GE2. (Para la diferencia de porcentajes de respuestas erróneas entre estos últimos, $\mathrm{z}=1,08, \mathrm{p}<0,28^{2}$.)

\section{FIGURA 3}

Porcentaje de estudiantes que piensan que es teóricamente posible lograr $100 \%$ de rendimiento.

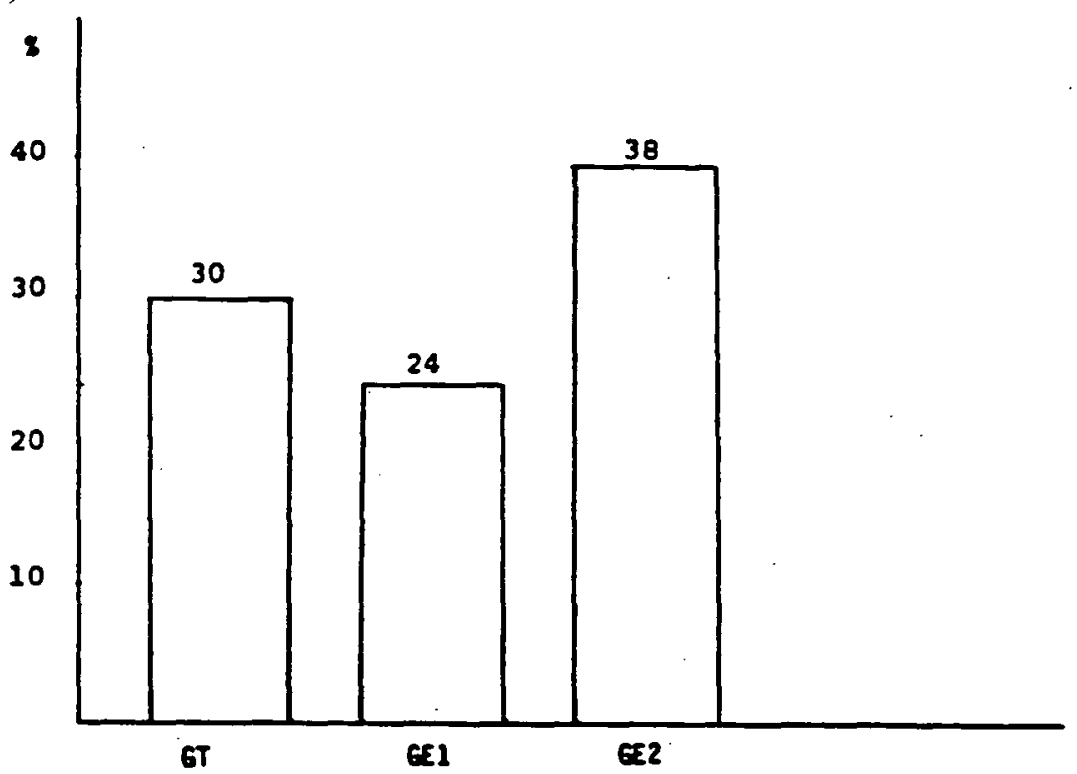

Una posible interpretación de estas respuestas es que, de acuerdo con el sentido común, se puede entender que un enunciado teórico es un enunciado hipotético o especulativo. La teoría en que parecen pensar los estudiantes no es la teoría científica, la teoría de la termodinámica, sino la «teoría» del sentido común. 
b) «Conservación del calor».

Algunos estudiantes mantienen una concepción del calor como algo que se conserva en el funcionamiento de una máquina térmica (con cierto parecido a la idea del funcionamiento de una máquina de vapor que tenía Carnot [1824]). El calor, en caso de que no se ceda, «se queda» en la máquina, aunque ésta esté realizando un trabajo. No parece manejarse en este contexto el principio de equivalencia entre el calor y el trabajo. Además, un número de alumnos parece considerar que el papel del foco frío es el de posibilitar la refrigeración de la máquina y no una necesidad ineludible impuesta por el Segundo Principio de la Termodinámica. Si se suprime, la máquina seguirá funcionando, pero se calentará cada vez más.

En la prueba B se incluyó una pregunta con la que, mediante dos formulaciones diferentes, se pretendía indagar sobre estas concepciones.

Pregunta B1 (versión A).

Si una máquina térmica convirtiese todo el calor en trabajo, absorbiendo el calor de un solo foco, al no poder disipar el calor por la inexistencia de foco frío se calentará progresivamente y terminará fundiéndose.

$\begin{array}{ccc}\text { Verdad } & \text { Mentira } & \text { No sé } \\ () & (1) & (1)\end{array}$

Explicación:

[Es verdad] porque si no tiene foco frío la temperatura irá subiendo constantemente hasta fundir la máquina, puede ser por el rozamiento o por el calor que se produce dentro de la máquina (GE1, B, 2).

[Es verdad] porque para que una máquina térmica funcione es necesario que haya un foco frío y otro caliente al no existir esto, no se puede refrigerar por lo que estallará (GT, B, 23).

Debe notarse que la necesidad de refrigeración para que funcione una máquina térmica, como el motor de un automóvil, es una concepción próxima al sentido común. Parece tener preeminencia, en las contestaciones que se acaban de citar, sobre otras ideas introducidas en la clase de Física como la equivalencia entre calor y trabajo.

No hay diferencias significativas $(p<0,05)$ en los porcentajes de respuestas erróneas en los dos grupos GT y GE1. Las respuestas incorrectas desaparecen casi totalmente en el grupo universitario que participó en este trabajo.

TABLA II

Contestaciones a la pregunta sobre el calentamiento de una máquina que transforma todo el calor en trabajo. (Prg. B1, versión A).

Contestaciones:

Correcta: En caso de que todo el calor se transforme en trabajo no se calentaría

Errónea: se calentaría NC 
Los errores son todavía más numerosos en una versión ligeramente diferente de la pregunta, utilizada con los alumnos del GE2.

Pregunta B1 (versión B).

Una máquina térmica, $A$, que funciona cíclicamente, absorbe $100 \mathrm{ca}$ lorías del foco caliente y cede 50 al foco frío. Una segunda máquina, B, también cíclica, absorbe las mismas calorías del mismo foco caliente, pero cede menos calorías al mismo foco frío. Esta máquina $\mathrm{B}$ siempre se calentará más que la $A$, y cuantas menos calorías ceda al foco frío, más se calentará.

$\begin{array}{ccc}\text { Verdad } & \text { Mentira } & \text { No sé } \\ (j) & (1) & (\quad)\end{array}$

Explicación:

[Es verdad] si cogiendo A y B el mismo calor una cede más que la otra es porque naturalmente una conserva más calor que la otra $\mathrm{y}$, por lo tanto, se calentará más (GE2, B, 5).

[Es verdad] Al ceder pocas calorías al foco frío, éstas se quedan en la máquina y al irse almacenando se va calentando más. (GE2, $\mathrm{B}, 26$ ).

[Es verdad] Porque el calor que coge si no lo cede, por la ley de conservación de la energía, se lo queda ella. (GE2, B, 24).

TABLA III

Respuestas sobre el calentamiento relativo de una máquina que cede menos calor que otra (Prg. B1, versión A).

\begin{tabular}{lc}
\hline Grupo & GE2 \\
\hline Contestaciones & \\
Correcta: la diferencia calor ab- & \\
sorbido - calor cedido se con- & 16 \\
vierte en trabajo & 10 \\
Errónea: cuanto menos calor se & 3 \\
ceda más se calentará & 29 \\
NC & 29 \\
Total &
\end{tabular}

Estas respuestas ponen de manifiesto la utilidad limitada que tienen para los alumnos principios como el de la equivalencia del calor y el trabajo. Los alumnos no piensan fácilmente en la posibilidad de que «el calor que coge si no lo cede» pueda convertirse en trabajo. Les resulta más inteligible el hecho de que se «quede» en la máquina como aparentemente sucede en el caso de un motor de automóvil al que no le funcione la refrigeración.

\section{II) Repetición de errores históricos}

Cuando los estudiantes tienen que usar conceptos que se les presentan en su forma final, como sucede en los materiales tradicionales, sin haber tenido oportunidad de criticar concepciones alternativas, repiten en ocasiones los mismos errores que se han cometido a lo largo de la historia. 
a) «Cálculo del rendimiento en parte de un ciclo».

El rendimiento de una máquina térmica debe calcularse. a lo largo de un proceso cerrado para asegurar que la sustancia de trabajo se encuentra finalmente en las mismas condiciones que al principio y, por tanto, que no existe variación en su energía interna. En el siglo XVIII se cometieron errores en el cálculo del rendimiento por no tener en cuenta esta condición. Algunos estudiantes repiten esta equivocación al usar solamente parte del ciclo de Carnot para averiguar el rendimiento de una máquina térmica. Calculan el rendimiento como un cociente entre el trabajo hecho por el sistema y el calor proporcionado al sistema, sin contar el trabajo que hay que realizar sobre la sustancia de trabajo para devolverla a su estado inicial.

En la Tabla 4 aparecen las respuestas a la pregunta B7:

Pregunta B7:

El rendimiento $(\eta=W / Q)$ del ciclo de Carnot de la figura puede calcularse dividiendo el trabajo efectuado por el sistema sobre el exterior (Wabc) por el calor proporcionado a la sustancia de trabajo (Qab).

Verdad

Mentira

No sé

( )

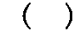

( )

TABLA IV

Contestaciones a la pregunta sobre el cálculo del rendimiento en parte de un ciclo de Carnot (Preg. B7).

\begin{tabular}{lcccc}
\hline Grupos & GT & GE1 & GE2 & GU \\
\hline Contestaciones & & & & \\
Correcta: No puede calcu- & & & & \\
larse (faltan datos) & 8 & 8 & 5 & 2 \\
No puede calcularse (no se & & & 6 & 4 \\
dice por qué) & 2 & 9 & 10 & 18 \\
Errónea: Puede calcularse & 5 & 5 & 8 & 9 \\
NC & 14 & 38 & 29 & 33 \\
Total & 29 & &
\end{tabular}

Según se observa en la tabla, el número de respuestas erróneas es máximo en el GU, tanto en términos relativos como absolutos. Sin embargo, muchos de los alumnos de este grupo que dicen que puede calcularse el rendimiento dividiendo el trabajo efectuado sobre el exterior (y no el trabajo neto) entre el calor-suministrado, al proporcionar la explicación dan fórmulas correctas para el cálculo del rendimiento (por ejemplo: $\mathbf{n}=1-\mathrm{Qcd} / \mathrm{Qab}$ ). El que, sin embargo, no comprendan el error en el enunciado de la pregunta, puede interpretarse como efecto de la superficialidad con que procesan la información que se les presenta, producto, a su vez, de la ausencia de inclusores estables. 
Porcentaje de estudiantes que calculan el rendimiento de una máquina de Carnot sobre parte del ciclo.

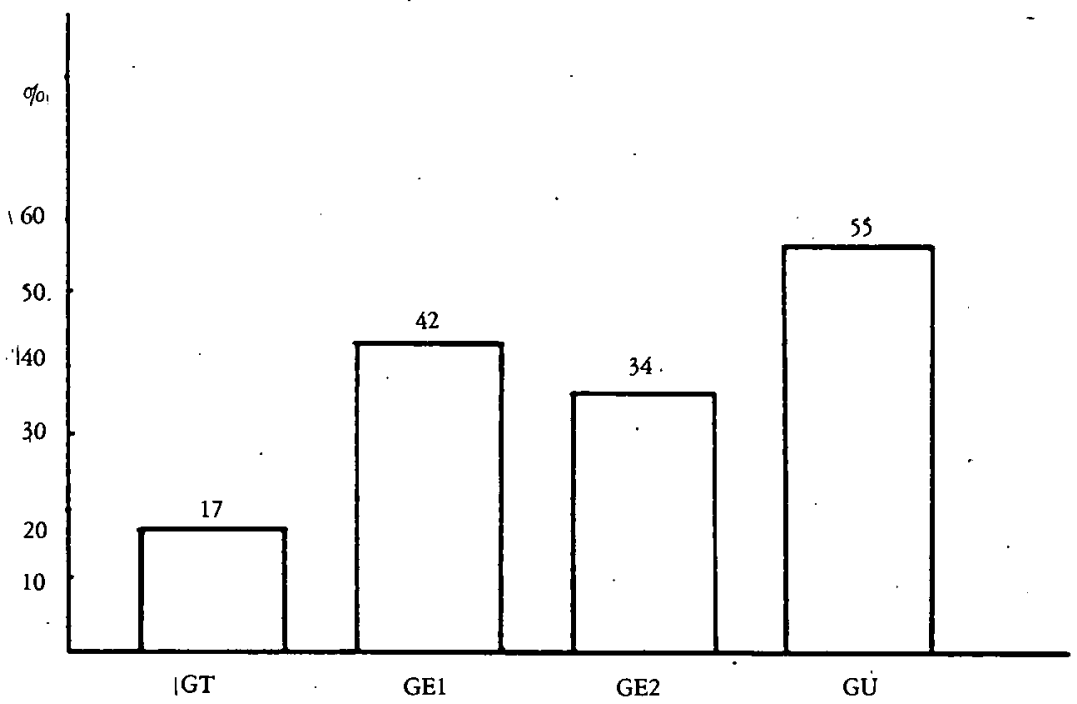

b) «Influencia de la sustancia de trabajo».

De la misma forma que históricamente hubo intentos de mejorar el rendimiento de las máquinas térmicas usando sustancias de trabajo con presiones de vapor altas (Payen, 1976, pág. 127) los alumnos reproducen esta clase de errores y creen que el tipo de sustancia de trabajo es importante para obtener más o menos rendimiento en una máquina que trabaje de acuerdo con el ciclo de Carnot. En la Tabla 5 se recogen las respuestas a la pregunta $\mathrm{B} 6$ :

Pregunta B6:

Una máquina de Carnot, funcionando entre dos temperaturas determinadas, tiene el máximo rendimiento posible. Ese máximo rendimiento será diferente dependiendo de la sustancia de trabajo (aire, vapor de $\mathrm{H}_{2} \mathrm{O}$, vapor de alcohol...) que utilice la máquina de Carnot.

$\begin{array}{ccc}\text { Verdad } & \text { Mentira } & \text { No sé } \\ (1) & () & (1)\end{array}$

TABLA V

Contestaciones a la pregunta sobre la posibilidad de que el rendimiento de una máquina de Carnot varie con la sustancia utilizada.

\begin{tabular}{lcccc}
\hline Grupos & GT & GE1 & GE2 & GU \\
\hline Constestaciones & & & & \\
Correcta: No Depende de la & & & & \\
sustancia & 14 & 27 & 24 & 20 \\
Errónea: Depende de la & 11 & 1 & 4 & 11 \\
sustancia & 4 & 0 & 1 & 2 \\
NC & 29 & 38 & 29 & 33 \\
Total & & & & \\
\hline
\end{tabular}


Las diferencias en los porcentajes de respuestas correctas entre GE2 y GT $(z=2,49 ; p<0,04)$ y entre GE1 y GT $(z=1,64 ; p<0,05)$ son significativas (pruebas de una sola cola).

\section{Figura 5}

Porcentajes de estudiantes que piensan que el tipo de sustancia de trabajo es importante para conseguir el rendimiento máximo en un ciclo de Carnot.

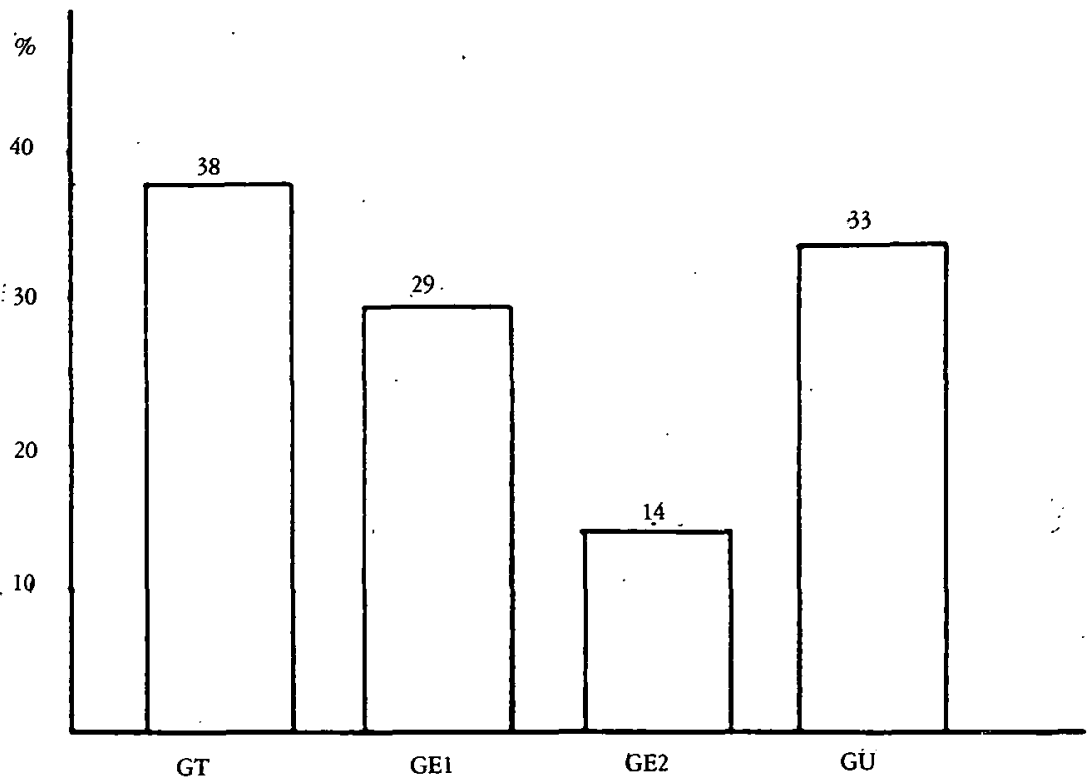

De los datos anteriores puede concluirse que muchos alumnos se enfrentan con los problemas científicos cometiendo algunos errores que cometieron los científicos a lo largo de la historia. A partir de los resultados obtenidos no puede concluirse que los materiales experimentales hayan tenido efectividad en todos los casos para paliar este problema.

\section{III) El aprendizaje de la segunda ley}

El recuerdo del enunciado de Kelvin-Planck de la Segunda Ley, des pués de 9 semanas, se tomó como medida de la significatividad de su aprendizaje. Se esperaba que la organización experimental (proble$\mathrm{ma} /$ solución) tuviese un efecto beneficioso.

En la Tabla 6 se recogen las contestaciones a la pregunta en que se solicitaba el enunciado de Kelvin-Planck. Se comparan los resultados de la primera prueba, realizada inmediatamente después de la terminación del período de aprendizaje, con los de la segunda, realizada 9 semanas después. Se recoge el número de alumnos que dan respuestas correctas tanto en la prueba $\mathrm{A}$ como en la prueba $\mathrm{B}$. Se recoge también el número de alumnos que pasan de dar una respuesta correcta (C) en la prueba $A$, a dar una respuesta errónea o no contestar en la prueba $\mathrm{B}(\mathrm{E} / \mathrm{NC})$. En el apartado «otras» se clasifican los restantes ca$\operatorname{sos}(\mathrm{E} / \mathrm{NC} \rightarrow \mathrm{E} / \mathrm{NC}, \mathrm{E} / \mathrm{NC} \rightarrow \mathrm{C})$. 


\begin{tabular}{lccc}
\hline Grupos & GT & GE1 & GE2 \\
\hline Contestaciones & & & \\
Recuerdan $\mathrm{C} \rightarrow \mathrm{C}$ & 11 & 23 & 5 \\
Olvidan $\rightarrow \mathrm{E} / \mathrm{NC}$ & 13 & 9 & 7 \\
Otras E $/ \mathrm{NC} \rightarrow \mathrm{E} / \mathrm{NC}, \mathrm{E} / \mathrm{NC} \rightarrow$ & 6 & 6 & 17 \\
$\quad \mathrm{C}$ & 6 & 38 & 29 \\
Total & 29 & & \\
\hline
\end{tabular}

Existen diferencias significativas entre GE1 y GE2 en los porcentajes de alumnos que enuncian correctamente la segunda ley en la prueba A $(z=2,64 ; p<0,008$, para una prueba de dos colas). Las diferencias entre los grupos GE1 y GE2, en éste, como en otros casos, pueden ser debidas tanto a la influencia del profesor como a las diferentes características de los alumnos.

Sólo hay diferencias significativas entre los grupos GE1 y GT, en cuanto a porcentajes de alumnos que recuerdan correctamente la segunda ley $(z=1,91 ; p<0,028)$. Los alumnos del GE2, además de haberla aprendido peor que los del GT, no la recuerdan mejor al cabo de 9 semanas.

En la Figura 6 se representan los porcentajes de alumnos que habiendo formulado correctamente la Segunda Ley en la prueba A la recuerdan y formulan correctamente en la prueba B. La diferencia positiva en el recuerdo para el GE1 puede explicarse porque, en contra de

\section{FIGURA 6}

Porcentajes de alumnos que recuerdan correctamente la $2^{a}$ Ley en la prueba $B$, calculados sobre aquellos que la habian formulado correctamente en la prueba $A$, 9 semanas antes.

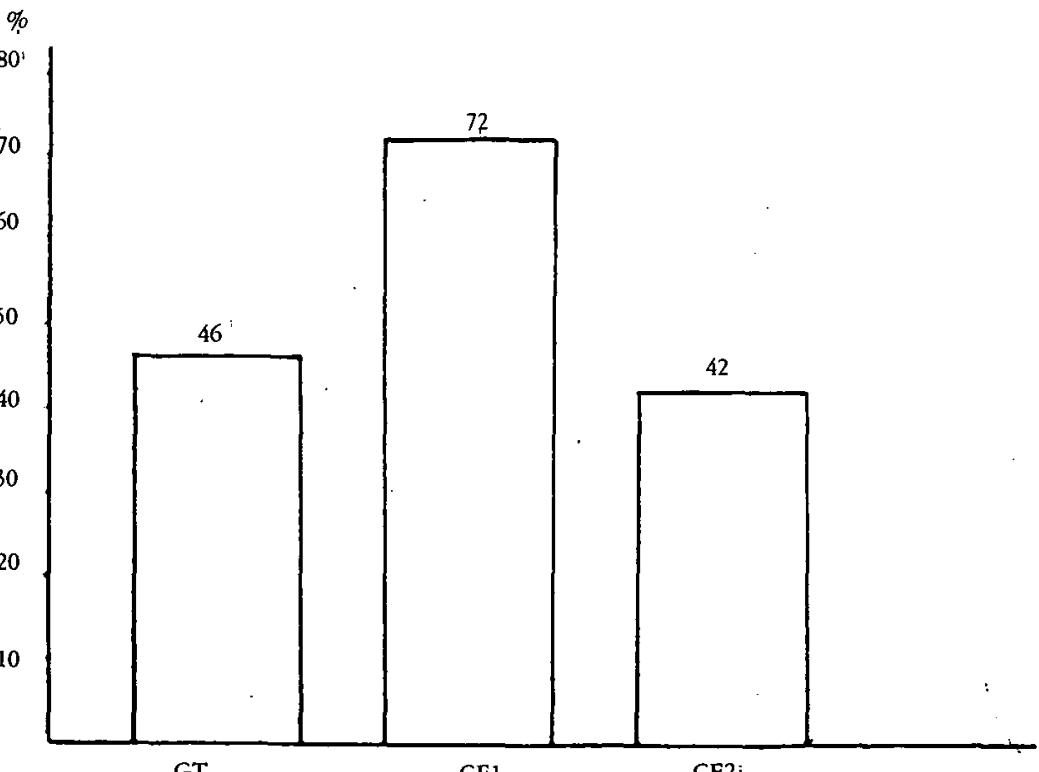


las instrucciones del equipo investigador, los alumnos de este grupo pudieron tener noticia de la celebración de la prueba $\mathrm{B}$ con anterioridad. Es muy probable, por tanto, que repasaran la materia poco anteś del examen.

IV) Dificultades en el aprendizaje de la estructura de alto nivel de los materiales

Los materiales experimentales intentaban mejorar el significado potencial de los conceptos presentándolos dentro de una estructura general que ayudase a ver la razón de ser para su aparición. La Segunda Ley, en particular, se presentaba como respuesta al problema del rendimiento de las máquinas térmicas ( $¿$ se puede aumentar ese rendimiento sin límite?).

En la prueba $\mathrm{B}$, se incluyó la siguiente pregunta de respuesta abierta:

B III. A continuación, están escritas cuatro ideas de las que has encontrado en el tema de la segunda ley de la Termodinámica. Escribe, por favor, una explicación que relacione estas cuatro ideas. Ten en cuenta que no se piden explicaciones aisladas de cada uno de los conceptos sino una explicación que los relacione entre sí.

Te sugerimos que la explicación ocupe al menos una cara completa del folio.

Primera idea: Rendimiento de las máquinas térmicas (Rendt).

Segunda idea: Proceso reversible (Rev).

Tercera idea: Ciclo de Carnot (Car).

Cuarta idea: Segunda ley de la Termodinámica según Kelvin (Kel). Explicación:

Se buscaron cinco posibles relaciones entre pares de conceptos (véase Fig. 7) en las contestaciones escritas. Para unificar la interpretación de las respuestas de los estudiantes, dos jueces las analizaron por separado utilizando criterios previamente definidos. Se consiguió un 94 por 100 de acuerdo en las relaciones identificadas.

Figura 7

Relaciones entre pares de conceptos que se buscaron en las respuestas escritas de los estudiantes.

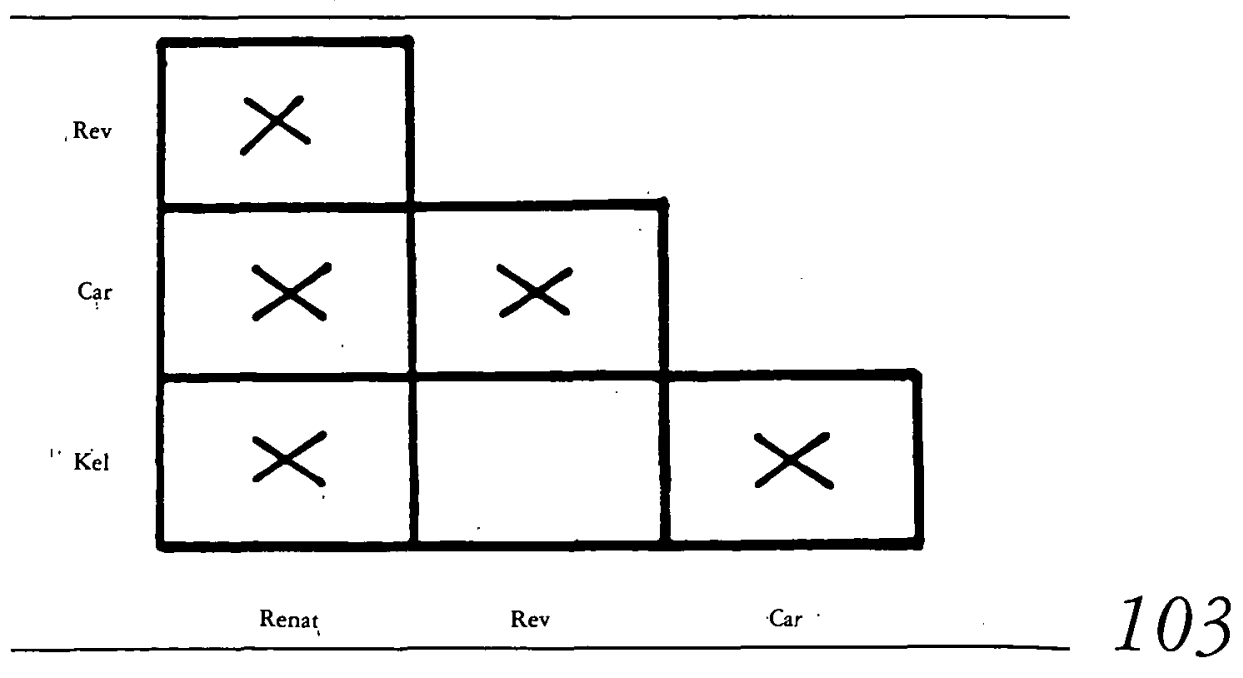


En la Tabla 7 se clasifican los estudiantes según el número de relaciones correctas establecidas. Se encuentra que el 24 por 100 de los estudiantes en el GE2, el 39 por 100 en el GE1, y el 28 por 100 en el GT son incapaces de establecer relación alguna entre los cuatro conceptos y principios que se proporcionaban en la prueba. El número de

TABLA VII

Alumnos según el número de relaciones establecidas correctamente entre los cuatro conceptos y principios proporcionados en la prueba BIII.

\begin{tabular}{|c|c|c|c|}
\hline Grupo & GT & GE1 & GE2 \\
\hline \multicolumn{4}{|c|}{$\begin{array}{l}\text { Número de relaciones } \\
\text { correctas }\end{array}$} \\
\hline 0 & 8 & 15 & 7 \\
\hline 1 & 11 & 14 & 10 \\
\hline 2 & 8 & 5 & 6 \\
\hline 3 & 2 & 3 & 5 \\
\hline 4 & - & 1 & 1 \\
\hline 5 & - & - & - \\
\hline Total & 29 & 38 & 29 \\
\hline
\end{tabular}

estudiantes que recuerda más de dos relaciones correctas se representa en la Figura 8. Ninguno de los estudiantes, en cualquier grupo, explicó correctamente la relación entre el ciclo de Carnot o el teorema de Carnot y la segunda ley de la Termodinámica aun cuando ésta era una relación fundamental para comprender la necesidad de introducir la Segunda Ley en los materiales experimentales.

Figura 8

Porcentajes de estudiantes que establecen más de dos relaciones correctas en sus respuestas a la pregunta $B$ III.

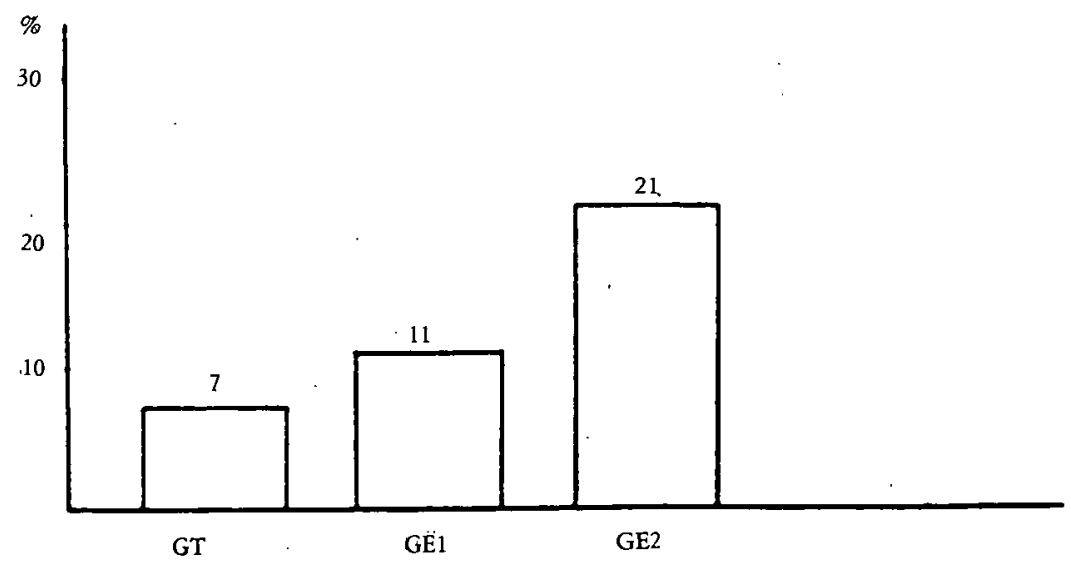

Los datos en este apartado sugieren que los alumnos usan solamente conceptos a un bajo nivel de generalidad, de manera que aprenden las distintas partes del contenido conceptual de manera inconexa, sin captar la macroestructura del texto. La efectividad de una estructuración conceptual general con mayor significado potencial, se ve.de esta forma muy limitada por la incapacidad de los alumnos para captar la organización general del contenido. 


\section{Conclusiones}

A partir de los datos anteriores, se pueden obtener tres grupos de conclusiones.

En primer lugar, debe destacarse, una vez más, el papel de las preconcepciones de los estudiantes en el aprendizaje de los conceptos físicos. Estas ideas interfieren en la enseñanza de las ciencias desde el nivel primario hasta los inicios del terciario, según refleja este estudio.

La recomendación práctica que se sigue, es la necesidad de identificar las preconcepciones de los alumnos y tenerlas en cuenta para evitar interferencias perjudiciales en el aprendizaje de nuevos conceptos. Ejemplos de estas interferencias, en el área que acabamos de considerar, son las relacionadas con los significados de «imposibilidad teoŕica y práctica de obtener un rendimiento del 100 por 100» o «transformación de calor en trabajo». El profesor debe ayudar a que el alumno discrimine entre el significado científico de los términos anteriores y posibles interpretaciones de «sentido común».

En segundo lugar, en lo que respecta al aprendizaje significativo, la hipótesis que se ha mantenido en este trabajo es que el aprendizaje de conceptos y principios dentro de la organización experimental y, en especial, el aprendizaje significativo de la Segunda Ley, dependían fuertemente de la comprensión de la estructura problema-solución del contenido. Sin embargo, los alumnos tienen grandes dificultades para comprender la macroestructura del texto; por tanto, no es de extrañar que las mejoras en el aprendizaje de la Segunda Ley sean pequeñas. Esta interpretación, acorde con conclusiones obtenidas por otros investigadores en este área (Sherris y Kahle, 1984), sugiere la necesidad de tratamientos más fuertes (Eylon y Reif, 1984), en donde se indiquen explícitamente las relaciones de alto nivel, si se quiere que los estudiantes aprendan la estructura conceptual tal como se intenta. Una vez asegurado el aprendizaje de las relaciones de alto nivel, podrían investigarse las diferencias entre estructuraciones alternativas.

Otro factor que podría explicar las deficiencias observadas en el aprendizaje significativo es el corto tiempo que duró el tratamiento. Es posible que en un período tan corto no se pueda conseguir una disposición para el aprendizaje significativo en estudiantes que están acostumbrados al aprendizaje memorístico de la ciencia (Novak y Gowin, 1984, pág. 160).

La disposición para el aprendizaje significativo de la ciencia, traducida en una mayor o menor profundidad de procesamiento de la información que le presentábamos a los alumnos, apareció como uno de los factores decisivos para el aprendizaje de cualquier organización conceptual de los materiales de enseñanza. El desarrollo de esta disposición es una tarea que, en nuestra opinión, merece la mayor prioridad, tanto por parte de profesores como de investigadores en didáctica de las ciencias.

En tercer lugar, se constata que los estudiantes repiten errores superados a lo largo de la historia de la ciencia cuando se le presentan los conceptos y principios científicos como resultados finales. Ello pone de manifiesto que conceptos y principios expuestos de esta forma distan mucho de ser obvios para los alumnos. Enfrentados a un proble$\mathrm{ma}$, los alumnos recurren en ocasiones a interpretaciones vigentes en tiempos pasados, y ya descartadas. La enseñanza actual de las ciencias no se suele ocupar de la crítica de estas alternativas confiando, quizá, 
en la «obviedad» de las soluciones, aceptadas actualmente, que se presentan a los alumnos.

Debe apuntarse finalmente que la naturaleza del trabajo realizado puede explicar, por una parte, que se hayan obtenido algunos resultados dispares entre ambos grupos experimentales, y por otra, que haya habido solamente mejoras discretas del aprendizaje de conceptos y principios debidas a la estructura evolutiva de los materiales experimentales. En un estudio exploratorio, como éste, no se controlan adecuadamente todas las variables que pueden intervenir en el aprendizaje. Las características y conocimientos de los alumnos, las características del profesor en cada uno de los grupos o las orientaciones que dan a los alumnos sobre el uso de los materiales, pueden influir de manera incontrolada en los resultados. En cualquier caso, lo que sí parecen indicar los datos que se han presentado, es que la estructura general del contenido conceptual en las lecciones de ciencias y el carácter «estático» del conocimiento conceptual incluido en ellas, son factores con influencia en el aprendizaje significativo de la ciencia. Se han identificado problemas de aprendizaje que aparecen en torno a ellos y, por tanto, parecen merecedores de la atención de investigadores y profesores de estas materias.

\section{Notas}

1 Lakatos se refiere a un fenómeno similar en el área de las matemáticas, en donde se oculta al alumno la naturaleza de los conceptos matemáticos «generados por las pruebas» (Lakatos, 1972, pág. 144).

2 z se calcula con la corrección de discontinuidad (Guilford, 1973, pág. 164).

\section{Resumen}

El curriculum de ciencias, especialmente en los niveles secundario y terciario, ba sido estructurado tradicionalmente de modo que refleja una visión estática del conocimiento científico. En este trabajo, se exploran las limitaciones que tiene tal estructuración del contenido conceptual, en el aprendizaje significativo de los alumnos. Se enseñó una lección sobre la Segunda Ley de la Termodinámica a alumnos de tercer curso de bachillerato, usando estructuraciones conceptuales alternativas, "estática" $y$ "evolutiva". Las conclusiones que se abtienen ponen de manifiesto, por una parte, el efecto sobre el aprendizaje de las preconcepciones de los alumnos $y$, por otra, la influencia de la organización conceptual general de los materiales de enseñanza.

\section{Summary}

Science curricula, specially at the secondary and tertiary levels, has been traditionally structured reflecting a static picture of scientific knowledge. This is an exploraty work in which the limitations of such a view, regarding meaningful learning, are studied. A lesson on the Second Law of Thermodynamics was taught using alternative conceptual organizations, "static" and "evolutionary". The results show, on the one hand, the effects of students' misconceptions on learning and, on the other, the influence of the general conceptual structure of the teaching materials.

\section{Résumé}

Le curriculum des sciences, et particulièrement dans les niveaux secondaire et universitaire, a toujours été structuré de façon à projeter une conception statique des connaissances scientifiques. Dans ce travail on explore les limites que possède ce type de structuration du contenu conceptuel dans le cadre de l'apprentissage significatif de l'élève. On a enseigné aux élèves de troisième cours du niveau secondaire une leçon versant sur la Seconde Loi de la Thermodinamique en utilisant différentes alternatives de structu- 
ration des concepts: statique et évolutive. Les conclusions ici présentées montrent d'un cóté l'éffet des preconceptions sur l'apprentissage et, d'autre part, l'influence que sur celui-ci exerce l'organisation générale des concepts qu'on trouve dans les matériels d'enseignement.

\section{Referencias}

Ausubel, D. P.; NovaK, J. D., y Hanesian, H.: Educational Psychology. Nueva York: Holt, Rinehart \& Winston, 1978. (Existe traducción al castellano: Editorial Trillas, 1983.)

CARNot, S.: Réflexions sur la puissance motrice du feu. París: Chez Bachelir, 1824. (Reimpresión con notas críticas de R. Fox, París: Vrin, 1978.)

ElKANA, Y.: «Science, Philosophy of Science and Science Teaching». Educational Philosophy and Theory, $1970,2,15-35$.

EYLON, B., y ReIF, F.: «Effects of Knowledge organization on Task Performance». Cognition and Instruction, 1984, I, 1, 5-44.

GUILFORD, P., y Fruchter, B.: Fundamental Statistics in Pscybalogy and Education. Tokio: Mc GrawHill, 1973. 5.a edición.

Halliday, D., y ResNick, R.: Física. Méjico: C.E.C.S.A., 1977.

Lakatos, I.: Proffs and Refutations. Cambridge: Cambridge University Press, 1976. (Existe traducción al castellano: Alianza Editorial, 1978.)

MYER, Bonnie J. F., y FreEdLE, Roy O.: «Effects of Discourse Type on Recall». American Educational Research Journal. Spring. Vol. 21, 1984, págs. 121-143.

NovaK, J. D., y GowIN, D. B.: Learning How to Learn. Cambridge: Cambridge University Press, 1984.

PAYEN, J.: «La pratique des machines a vapeur au temps de Carnot», en Sadi Carnot et l'essor de la thermodynamique. parís: Editions du CNRS, 1976.

ReICHENBACH, H.: Experience and prediction. Chicago: The University of Chicago Press, 1938.

SCHAB, J., y BRANDwEIn, P. F.: The Teaching of Science. Cambridge: Cambridge University Press, 1962.

SHERRIS, J. D., y KAHLE, J. B.: «The Effects of Instructional organization and Locus of Control orientation on meaningful learning in High School Biology Students». Journal of Research in Science Teaching, 1984, 21 (1), 83-94.

Toulmin, S.: Human Understanding. Vol. I. The Collective use and Evolution of Concepts. Princeton; N. J.: Princeton University Press, 1972. (Existe traducción al castellano: Alianza Editorial, 1971.) 